\title{
On Huygens' Principle for the Hodge-de Rham Equations with Lorentzian Gauge
}

\author{
V. Wünsch
}

Dedicated to the memory of Professor Dr. Paul Günther

\begin{abstract}
In an arbitrary curved space-time the Hodge-de Rham equations with Lorentzian gauge are studied. Using the spinor calculus and propositions on the curvature tensors, especially on Hall's canonical forms of Ricci tensors, some properties of the tail terms with respect to second order differential operators are proved. Finally, all Huygens' operators are explicitly determined.
\end{abstract}

Keywords: Hodge-de Rham equations, Lorentzian gauge, curved space-times, Huygens'principle, tail terms, plane wave metrics

AMS subject classification: $58 \mathrm{G} 16,83 \mathrm{C}, 35 \mathrm{~B} 30$

\section{Introduction}

In a four-dimensional pseudo-Riemannian manifold $(M, g)$ with a smooth metric of Lorentzian signature the Hodge-de Rham equations for $p$-forms with Lorentzian gauge

$$
\begin{aligned}
& \Delta u=w \\
& \delta w=0
\end{aligned} \quad\left(u, w \in \Lambda^{p}, p=1,2\right)
$$

are considered, where $\Delta=-(d \delta+\delta d)$ denotes the Hodge-de Rham operator (see [2, 5, $12,15,18,19]), d$ the exterior derivative and $\delta$ the co-derivative. The equations (1.1) are of physical interest. Especially, if $u \in \Lambda^{1}$ is the electromagnetic vector potential and the source $w$ represents a charged particle moving along a world line, then the divergence of $w$ must vanish (see, e.g., F. G. Friedlander [1]).

For the equations (1.1) Huygens' principle (in the sense of Hadamard's "minor premise") is valid if the solution of Cauchy's initial value problem in a sufficiently small neighbourhood of the initial space-like surface $F$ depends only on the Cauchy data in an arbitrarily small neighbourhood of the intersection of the past semi-null cone with $F$ (see $[2,5,7,13,18,19]$ ). Only if Huygens' principle is valid, then the wave propagation

V. Wünsch: Friedrich-Schiller-Universität, Mathematisches Institut, Ernst-Abbe-Platz 4, D 07743 Jena

I wish to express my deepest respect and sincere gratidude to my academic teacher Professor $P$. Günther, who drew my attention to Hadamard's problem of the Huygens' principle and always generously supported my scientific efforts. 
is free of tails (see $[2,5,7]$ ), i.e. the solution depends only on the source distribution on the past null cone of the field point and not on the sources inside the cone.

The present paper is motivated by earlier investigations on Huygens' principle for the usual Hodge-de Rham equations (without Lorentzian gauge) (see $[5,18,19])$.

The main result in this paper reads as follows.

Theorem 1.1.

(i) The equations

$$
\begin{aligned}
\Delta u & =w \\
\delta w & =0
\end{aligned} \quad\left(u, w \in \Lambda^{1}\right)
$$

satisfy Huygens' principle if and only if $g$ is either'a plane wave metric or a metric with $C_{a b c d}=0$ and $R_{a b}=\frac{1}{4} R g_{a b}$.

(ii) The equations

$$
\begin{aligned}
\Delta u & =w \\
\delta w & =0
\end{aligned} \quad\left(u, w \in \Lambda^{2}\right)
$$

satisfy Huygens' principle if and only if $g$ is either a plane wave metric or a metric with $C_{a b c d}=0$ and $R\left(R_{a b}-\frac{1}{4} R g_{a b}\right)=0$.

The paper is organized as follows:

After some preliminaries we give in Section 3 some necessary and sufficient conditions for the validity of Huygens' principle for equations (1.1). We show relations for the tail terms with respect to some differential operators and determine the first coincidence values of the tail terms. In Section 4, the spinor calculus, Hall's canonical forms of the Ricci tensor, some properties of the curvature tensors, and the second coincidence value of the tail terms are used to prove Theorem 1.1 .

\section{Preliminaries}

Let $(M, g)$ be a space-time, i.e. a 4-manifold together with a smooth metric of Lorentzian signature, and $g_{a b}, g^{a b}, \nabla_{a}, R_{a b c d}, R_{a b}, R$ and $C_{a b c d}$ the local coordinates of the covariant and contravariant metric tensor, the Levi-Civita connection, the curvature tensor, the Ricci tensor, the scalar curvature and the Weyl curvature tensor, respectively. The signs of the curvature tensor and of the Ricci tensor are determined by the Ricci identity

$$
\nabla_{[a} \nabla_{b]} T_{c}=-\frac{1}{2} R_{a b c d} T^{d}
$$

and

$$
R_{a b}=g^{l k} R_{a l k b}
$$

respectively. $\Lambda^{p}$ denotes the space of $p$-forms of class $C^{\infty}$. On $\Lambda^{p}$ the exterior derivative $d$, the coderivative $\delta$ and $\Delta=-(d \delta+\delta d)$ are defined. The following relations are valid $(\sec [5,12])$ :

$$
\begin{aligned}
& (d u)_{a_{1} \cdots a_{p+1}}=\nabla_{\left[a_{1}\right.} u_{\left.a_{2} \cdots a_{p+1}\right]} . \\
& (\delta u)_{a_{1} \cdots a_{p-1}}=-p \nabla^{k} u_{k a_{1} \cdots a_{p-1}}
\end{aligned} \quad\left(u \in \Lambda^{p}\right)
$$


and

$$
\begin{aligned}
\left(L^{(2)} u\right)_{a_{1} a_{2}}:=(\Delta u)_{a_{1} a_{2}} & =\square u_{a_{1} a_{2}}-C_{a_{1} a_{2}}{ }^{c d} u_{c d}-\frac{1}{3} R u_{a_{1} a_{2}} \quad\left(u \in \Lambda^{2}\right) \\
\left(L^{(1)} u\right)_{a_{1}} & :=(\Delta u)_{a_{1}}=\square u_{a_{1}}-R_{a_{1}}{ }^{b} u_{b} \quad\left(u \in \Lambda^{1}\right) \\
L^{(0)} u & :=-(\delta d u)=\square u \quad\left(u \in \Lambda^{0}\right)
\end{aligned}
$$

where $\square=g^{a b} \nabla_{a} \nabla_{b}$. Because of the commutator relations (see [5: pp. 283])

$$
\delta L^{(p)}=L^{(p-1)} \delta \quad(p>0)
$$

the operator $L^{(p)}$ maps

$$
\Lambda_{\delta}^{p}=\left\{u \in \Lambda^{p}: \delta u=0\right\}
$$

into itself and the Hodge-de Rham equations (1.1) with Lorentzian gauge can be written as

$$
L^{(p)} u=w \quad\left(u, w \in \Lambda_{\delta}^{p}, p=1,2\right) .
$$

Let $M$ be a causal domain (see $[2,5])$ and $\Gamma(x, y)$ the square of geodesic distance of $x, y \in M$. For any fixed $y \in M$ the set $\{x \in M: \Gamma(x, y)>0\}$ decomposes naturally into the open subsets $D_{+}(y)$ and $D_{-}(y)$ called future and past of $y$, respectively. The characteristic semi-null cones $C_{ \pm}(y)$ are defined as the boundary sets of $D_{ \pm}(y)$, respectively. Then $D_{+}(y)$ consists of those points $x \in M$ for which the geodesic segment from $y$ to $x$ is causal and future-oriented. If this segment is a time like or a null line, then $x$ belongs to int $D_{+}(y)$ or $C_{+}(y)$, respectively.

Let $G_{ \pm}^{p}(y) \quad(p=0,1,2)$ be the fundamental solution of the operator $L^{(p)}$ and $T^{(p)}(\cdot, y)$ the tail term of $G_{ \pm}^{p}(y)$ with respect to $y$. Then the inclusion supp $G_{ \pm}^{p}(y) \subseteq$ $D_{ \pm}(y)$ holds (see $[2,5]$ ). The tail term is just the factor of the regular part of the corresponding fundamental solution. For $T^{(p)}$ there is an asymptotic expansion

$$
T^{(p)}(x, y) \sim \sum_{k=0}^{\infty} \frac{1}{2^{k} k !} U_{k+1}^{(p)}(x, y)(\Gamma(x, y))^{k}
$$

where the Hadamard coefficients $U_{k}^{(p)}$ are determined recursively by the transport equations (see $[2,5,13,19])$

$$
\nabla^{a} \Gamma \nabla_{a} U_{k}^{(p)}+\frac{1}{2}(\square \Gamma-8+4 k) U_{k}^{(p)}=-L^{(p)} U_{k-1}^{(p)} \quad(k \geq 0)
$$

with the initial conditions

$$
U_{-1}^{(p)} \equiv 0 \quad \text { and } \quad U_{0}^{(p)}(y, y)=I^{(p)}(y)
$$

where $I^{(p)}$ denotes the identity. ${ }^{1)}$ For a timelike separation of $x$ and $y, T^{(p)}(\cdot, y)$ is defined as the unique solution of the characteristic initial value problem

$$
\begin{gathered}
L^{(p)} T^{(p)}(\cdot, y)=0 \\
\left.T^{(p)}(x, y)\right|_{\Gamma=0}=0
\end{gathered}
$$

(see $[2,5,13,18,19])$.

1) The operator $L^{(p)}$ and all derivatives refer to $x$. 


\section{Huygens' principle}

From Günthers' investigations there follows (see [5: Chapter IV]):

Proposition 3.1.

(i) The Hodge-de Rham operator $L^{(p)}: \Lambda^{p} \rightarrow \Lambda^{p}$ is a Huygens'operator 2) if and only if

$$
T^{(p)}(x, y)=0
$$

for all $x$ and $y$.

(ii) The Hodge-de Rham operator with Lorentzian gauge $L^{(p)}: \Lambda_{\delta}^{p} \rightarrow \Lambda_{\delta}^{p}$ is a Huygens' operator ${ }^{3)}$ if and only if

$$
d_{(x)} T^{(p)}(x, y)=0
$$

for all $x$ and $y$.

In $[18,19]$ the following proposition was proved:

\section{Proposition 3.2.}

(i) $L^{(1)}: \Lambda^{1} \rightarrow \Lambda^{1}$ is a Huygeñs'operator if and only if $g$ is flat.

(ii) $L^{(2)}: \Lambda^{2} \rightarrow \Lambda^{2}$ is a Huygens' operator if and only if $C_{a b c d}=0$ and $R=0$.

Remark 3.1. Obviously, the operator $L^{(p)}: \Lambda_{\delta}^{p} \rightarrow \Lambda_{\delta}^{p}$ is a Huygens' one if $g$ is flat. In the following we are interested in the determination of all metrics for which $L^{(p)}: \Lambda_{\delta}^{p} \rightarrow \Lambda_{\delta}^{p}$ is a Huygens' operator.

The tail terms $T^{(p)}(x, y)$ satisfy the relations (see [5: p. 289])

$$
\delta_{(x)} T^{(p)}(x, y)=d_{(y)} T^{(p-1)}(x, y) \quad(p=1,2) .
$$

Corollary 3.1. From (3.2) it follows

$$
d_{(x)} d_{(y)} T^{(p-1)}(x, y)=0
$$

for all $x$ and $y$.

Proof. The relations (3.3), (2.10) and (3.2) imply

$$
d_{(x)} \delta_{(x)} T^{(p)}(x, y)=d_{(x)} d_{(y)} T^{(p-1)}(x, y)=-\delta_{(x)} d_{(x)} T^{(p)}(x, y)=0
$$

and thus the assertion is proved

\footnotetext{
2) I.e. Huygens' principle for the corresponding equation $L^{(p)} u=w\left(u, w \in \Lambda^{p}\right)$ is satisfied, see Section 1.

3) In this case $\left(\delta, L^{(p)}, I^{(p)}\right)$ is a Huygens' triple, see [5: pp. 249].
} 
Remark 3.2. The condition (3.4) is satisfied for $p=2$ if and only if the Maxwell equations

$$
\begin{aligned}
& d u=0 \\
& \delta u=w
\end{aligned} \quad\left(u \in \Lambda^{2}, w \in \Lambda^{1}\right)
$$

form a Huygens' system (see [5: p. 288]).

Remark 3.3. A metric $g$ is said to be plane wave if $d s^{2}=g_{a b} d x^{a} d x^{b}$ has the form

$$
d s^{2}=2 d x^{1} d x^{2}-\sum_{\alpha, \beta=3}^{4} a_{\alpha \beta}\left(x^{1}\right) d x^{\alpha} d x^{\beta}
$$

where the matrix $\left(a_{\alpha \beta}\right)$ is positive definite (see $\left.[2,4,5,17]\right)$.

The following result was proved in [5: pp. $683-685]$ :

Proposition 3.3. If $g$ is a plane wave metric, then $d_{(x)} T^{(p)}(x, y)=0(p=1,2)$.

The next step is the determination of $d_{(x)} T^{(p)}(y, y)$. For this purpose, for $u \in \Lambda^{p}$ we define

$$
C^{(p)} u:=L^{(p)} u-\square u \text {. }
$$

Then from $(2.3)-(2.5)$ it follows that

$$
\begin{aligned}
C^{(0)} u & =0, \quad\left(C^{(1)} u\right)_{a_{1}}=-R_{a_{1}}{ }^{b} u_{b} \\
\left(C^{(2)} u\right)_{a_{1} a_{2}} & =-C_{a_{1} a_{2}}{ }^{c d} u_{c d}-\frac{1}{3} R u_{a_{1} a_{2}}
\end{aligned}
$$

and for the corresponding Cotton invariants $\mathfrak{C}^{(p)}:=C^{(p)}+\frac{1}{6} R I^{(p)}$ (see $[2,5,18]$ ) we obtain

$$
\begin{aligned}
\mathfrak{C}^{(0)} & =\frac{1}{6} R \\
\mathfrak{C}_{a}^{(1) b} & =L_{a}^{b} \\
\mathfrak{C}_{a_{1} a_{2}}^{(2) b_{1} b_{2}} & =-C_{a_{1} a_{2}} b_{1} b_{2}-\frac{1}{6} R \delta_{\left[a_{1}\right.}^{b_{1}} \delta_{\left.a_{2}\right]}^{b_{2}}
\end{aligned}
$$

where $L_{a b}=-R_{a b}+\frac{1}{6} R g_{a b}$. The curvature operators $K_{a_{1} a_{2}}^{(p)}$ are defined by the Ricci identity (see $[5,18])$

$$
\nabla_{\left[a_{1}\right.} \nabla_{\left.a_{2}\right]} u=-\frac{1}{2} K_{a_{1} a_{2}}^{(p)} \cdot u \quad\left(u \in \Lambda^{p}\right)
$$

Consequently, because of (2.1)

$$
\begin{aligned}
K_{a_{1} a_{2}}^{(0)} & =0 \\
K_{a_{1} a_{2} c}^{(1) d} & =R_{a_{1} a_{2} c}{ }^{d} \\
K_{a_{1} a_{2} c_{1} c_{2}}^{(2)}{ }^{d_{1} d_{2}} & =R_{a_{a_{1} a_{2}}\left[c_{1}\right.}\left[d^{\left[d_{1}\right.} \delta_{\left.c_{2}\right]}{ }^{\left.d_{2}\right]} .\right.
\end{aligned}
$$


Under consideration of (2.8), for the coincidence values $T^{(p)}(y, y)$ and $\nabla_{i_{1}} T^{(p)}(y, y)$ we obtain (see [5: p. 576]) $\left.{ }^{4}\right)$

$$
\begin{aligned}
T^{(p)} & =U_{1}^{(p)}=-\frac{1}{2} \mathfrak{C}^{(p)} \\
\nabla_{i_{1}} T^{(p)} & =\nabla_{i_{1}} U_{1}^{(p)}=\frac{1}{12} \nabla^{a} K_{a i_{1}}^{(p)}-\frac{1}{4} \nabla_{i_{1}} \mathfrak{C}^{(p)}
\end{aligned}
$$

Hence, on account of (3.9) and (3.11)

$$
\begin{aligned}
\left(d_{(x)} T^{(1)}\right)_{\left[a_{1} a_{2}\right] \alpha}= & \frac{1}{12} \nabla^{a} R_{a\left[a_{1} a_{2}\right] \alpha}-\frac{1}{4} \nabla_{\left[a_{1}\right.} L_{\left.a_{2}\right] \alpha} \\
\left(d_{(x)} T^{(2)}\right)_{\left[a_{1} a_{2} a_{3} \mid \alpha_{1} \alpha_{2}\right.}= & {\left[\frac{1}{12} \nabla^{a} R_{a_{a_{1} a_{2} \alpha_{1}} g_{a_{3} \alpha_{2}}}\right.} \\
& \left.+\frac{1}{4} \nabla_{a_{1}}\left(C_{a_{2} a_{3} \alpha_{1} \alpha_{2}}+\frac{1}{6} R g_{a_{2} \alpha_{1}} g_{a_{3} \alpha_{2}}\right)\right]_{\left[a_{1} a_{2} a_{3}\right],\left[\alpha_{1} \alpha_{2}\right]} .
\end{aligned}
$$

Now an easy calculation leads to the equivalence relation

$$
d_{(x)} T^{(p)}=0 \quad \Longleftrightarrow \quad \nabla_{[a} R_{b] c}=0 \quad(p=1,2)
$$

and condition (3.2) implies the following

Proposition 3.4. For $L^{(p)}: \Lambda^{(p)} \rightarrow \Lambda^{p}$ to be a Huygens' operator the condition

$$
\nabla_{[a} R_{b] c}=0
$$

is necessary.

Remark 3.4. Obvously, (3.16) is equivalent to

$$
\begin{aligned}
\nabla_{\{a} L_{b] c}=\nabla^{k} C_{k c a b} & =0 \\
\nabla_{\alpha} R & =0 .
\end{aligned}
$$

A space-time $(M, g)$ with property $\nabla^{k} C_{k a b c}=0$ is called a $C$-space-time.

In $[21,22]$ the following result was proved (see Corollary 3.1 and Remark 3.2):

Proposition 3.5. The relation $d_{(x)} d_{(y)} T^{(1)}(x, y)=0$ and (3.17) imply that $g$ is conformally equivalent to a plane wave metric or to a flat metric.

There holds (see $[13,20-22\})$ :

Proposition 3.6. Assuming (3.17) every metric $g$, which is conformally equivalent to a plane wave metric, is a plane wave metric.

Now, the following lemma follows immediately from $T^{(0)}=-\frac{1}{12} R$ (see (3.9) and (3.12)) and the property $T^{(0)}(x, y)=T^{(0)}(y, x)$ (see $[2,5]$ ) by Taylor expansion of $T^{(0)}(x, y)$ in $x=y$ :

4) If we disregard in the derivatives of $T^{(p)}, U^{(p)}, \ldots$ the variables, then we stipulate their coincidence values. 
Lemma 3.1. In the case $R=$ const the condition $d_{(x)} d_{(y)} T^{(0)}(x, y)=0$ implies

$$
T S\left(\nabla_{i_{1}} \cdots \nabla_{i_{r}} T^{(0)}\right)=0 \quad(r>0)
$$

where $T S(T \ldots)$ denotes the trace-free symmetric part of the tensor $T \ldots$

Now we need the coincidence values $\nabla_{\left(i_{1}\right.} \nabla_{\left.i_{2}\right)} T^{(p)}$ under the condition $\nabla_{i_{1}} T^{(p)}=0$ $($ see $(3.16))$.

Lemma 3.2. Assuming (3.17) one has

$$
\begin{aligned}
\nabla_{\left(i_{1}\right.} \nabla_{\left.i_{2}\right)} T^{(p)}= & I_{i_{1} i_{2}}^{(p)}-\frac{1}{12} T S\left(R_{i_{1} i_{2}}\right) \mathfrak{C}^{(p)} \\
& -\frac{1}{6} T S\left(\nabla_{i_{1}} \nabla_{i_{2}} \mathfrak{C}^{(p)}\right)+\frac{1}{8} g_{i_{1} i_{2}} C^{(p)} \cdot \mathfrak{C}^{(p)}
\end{aligned}
$$

where

$$
I_{i_{1} i_{2}}^{(p)}=-\frac{1}{12}\left[\frac{1}{10} C^{a}{ }_{i_{1} i_{2}}{ }^{b} R_{a b} I^{(p)}+g^{a b} K_{i_{1} a}^{(p)} \cdot K_{i_{2} b}^{(p)}-\frac{1}{4} g_{i_{1} i_{2}} K_{a}^{(p) b} K_{b}^{(p) a}\right]
$$

is the moment of order 2 with respect to the operator $L^{*}:=\square-\frac{1}{6} R: \Lambda^{p} \rightarrow \Lambda^{p}$ (see $[5])$.

Proof. From (2.10) it follows that $\square T^{(p)}(\cdot, y)=-C^{(p)} T^{(p)}(\cdot, y)$. Consequently, because of (2.8)

$$
\nabla_{\left(i_{1}\right.} \nabla_{\left.i_{2}\right)} T^{(p)}=T S\left(\nabla_{i_{1}} \nabla_{i_{2}} U_{1}^{(p)}\right)-\frac{1}{4} g_{i_{1} i_{2}} C^{(p)} \cdot T^{(p)}
$$

Now, (2.9) implies (see $[5,18,19])$

$$
\begin{aligned}
T S\left(\nabla_{i_{1}} \nabla_{i_{2}} U_{1}^{(p)}\right)= & \frac{1}{9} T S\left(R_{i_{1} i_{2}}\right) U_{1}^{(p)} \\
& -\frac{1}{6} T S\left[\nabla_{i_{1}} \nabla_{i_{2}} L^{*} U_{0}^{(p)}+\mathfrak{C}^{(p)} \cdot \nabla_{i_{1}} \nabla_{i_{2}} U_{0}^{(p)}+\nabla_{i_{1}} \nabla_{i_{2}} \mathfrak{C}^{(p)}\right] .
\end{aligned}
$$

By virtue of

$$
\begin{aligned}
& T S\left(\nabla_{i_{1}} \nabla_{i_{2}} U_{0}^{(p)}\right)=\frac{1}{6} T S\left(R_{i_{1} i_{2}}\right) I^{(p)} \\
& L^{*} U_{0}^{(p)}=0, \quad \nabla_{i_{1}}\left(L^{*} U_{0}^{(p)}\right)=0 \\
& T S\left(\nabla_{i_{1}} \nabla_{i_{2}}\left[L^{*} U_{0}^{(p)}\right]\right)=-6 I_{i_{1} i_{2}}^{(p)}
\end{aligned}
$$

(see $[5,18,19])$ we obtain the assertion (3.19)

From (3.8), (3.9), (3.11) and (3.20) we obtain the following result: 
Corollary 3.2. One has

$$
\nabla_{\left(i_{1}\right.} \nabla_{\left.i_{2}\right)} T^{(0)}=-\frac{1}{120} C^{a}{ }_{i_{1} i_{2}}{ }^{b} R_{a b}-\frac{1}{72} R\left[T S\left(R_{i_{1} i_{2}}\right)\right]
$$

and, for $C_{a b c d}=0$,

$$
\begin{aligned}
\nabla_{\left(i_{1}\right.} \nabla_{\left.i_{2}\right)} T_{a}^{(1) \alpha}= & -\frac{1}{12} R_{\left(i_{1} k a\right.}{ }^{l} R_{\left.i_{2}\right)}{ }^{k} l^{\alpha}+\frac{1}{48} g_{i_{1} i_{2}} R_{k s a}{ }^{l} R^{k s_{l}{ }^{\alpha}} \\
& -\frac{1}{6} T S\left(\nabla_{i_{1}} \nabla_{i_{2}} L_{a}{ }^{\alpha}\right)-\frac{1}{12} T S\left(R_{i_{1} i_{2}}\right) L_{a}{ }^{\alpha}-\frac{1}{8} g_{i_{1} i_{2}} R_{a}{ }^{k} L_{k}{ }^{\alpha}
\end{aligned}
$$

and

$$
\begin{aligned}
\nabla_{\left(i_{1}\right.} \nabla_{\left.i_{2}\right)} T_{a_{1} a_{2}}^{(2) \alpha_{1} \alpha_{2}}= & -\frac{1}{12} R_{\left(i _ { 1 } k \left[a_{1}\right.\right.}{ }^{[c} \delta_{\left.a_{2}\right]}^{d]} R_{\left.i_{2}\right)}{ }_{c}{ }_{c}^{\left[\alpha_{1}\right.} \delta_{d}^{\left.\alpha_{2}\right]}+\frac{1}{72} \operatorname{RTS}\left(R_{i_{1} i_{2}}\right) \delta_{\left[a_{1}\right.}^{\left(\alpha_{1}\right.} \delta_{\left.a_{2}\right]}^{\left.\alpha_{2}\right]} \\
& +\frac{1}{48} g_{i_{1} i_{2}} R_{k s\left[a_{1}\right.}\left[{ }^{[} \delta_{a_{2}}^{d]} R^{k s}{ }_{c}{ }^{\left[\alpha_{1}\right.} \delta_{d}^{\left.\alpha_{2}\right]}+\frac{1}{144} g_{i_{1} i_{2}} \delta_{\left[a_{1}\right.}^{\alpha_{1}} \delta_{\left.a_{2}\right]}^{\alpha_{2}} R^{2} .\right.
\end{aligned}
$$

Proposition 3.7. If $R=0$ and $\nabla_{[a} R_{b] c}=0$, then the condition $d_{(x)} d_{(y)} T^{(0)}(x, y)=$ 0 implies that $g$ is conformally flat or a plane wave metric.

Proof. From (3.12) there follows $T^{(0)}=0$ and, by virtue of Lemma 3.1, the conditions

$$
T S\left(\nabla_{i_{1}} \cdots \nabla_{i_{r}} T^{(0)}\right)=0 \quad(0 \leq r \leq 6)
$$

imply the assertion (see $[21,22]$ )

The following two propositions were proved in [20].

Proposition 3.8. If the relations

$$
\nabla_{[a} R_{b] c}=0, \quad R \neq 0, \quad C_{a b c d} R^{b c}=\varepsilon R\left(R_{a d}-\frac{1}{4} R g_{a d}\right)
$$

hold with an $\varepsilon \in \mathbb{R} \backslash\left\{-\frac{1}{6}, 0, \frac{1}{3}\right\}$, then one has $T S\left(R_{a d}\right)=0$.

Proposition 3.9. If in a non-conformally flat Einstein space-time the relations

$$
T S\left(\nabla_{a} C_{b i_{1} i_{2}}{ }^{c} \nabla^{a} C^{b}{ }_{i_{3} i_{4} c}-\frac{\varepsilon}{24} R C_{a i_{1} i_{2}}{ }^{b} C^{a}{ }_{i_{3} i_{4} b}\right)=0
$$

hold with an $\varepsilon \in \mathbb{R}$ and $R \neq 0$, then $\varepsilon \in\{0,-26\}$.

Corollary 3.3. Assuming $R \neq 0$ and $\nabla_{[a} R_{b] c}=0$ the conditions (3.18) imply that $g$ is conformally flat.

Proof. The relations (3.25) with $\varepsilon=-\frac{5}{3}$ follow from (3.18) and (3.21). Consequently, because of Proposition 3.8, one has $T S\left(R_{a b}\right)=0$. Furthermore, under consideration of $U_{1}^{(0)}=-\frac{1}{12} R$ (see (3.12)), $T S\left(R_{a b}\right)=0$ and (see [16])

$$
\begin{array}{r}
\operatorname{TS}\left[\nabla_{i_{1}} \cdots \nabla_{i_{1}}(\square \Gamma-8)\right]=-16 T S\left(\nabla_{i_{1}} \cdots \nabla_{i_{4}} U_{0}^{(0)}\right) \\
\cdots \quad \therefore \quad=-\frac{16}{15} T S\left(C^{a}{ }_{i_{1} i_{2}}{ }^{b} C_{a i_{3} i_{4} b}\right)
\end{array}
$$


we obtain from (2.8) and (2.9)

$$
\begin{aligned}
T S\left(\nabla_{i_{1}} \cdots \nabla_{i_{4}} T^{(0)}=\right. & T S\left(\nabla_{i_{1}} \cdots \nabla_{i_{4}} U_{0}^{(0)}\right) \\
= & -\frac{1}{20} T S\left[\nabla_{i_{1}} \cdots \nabla_{i_{4}}(\square \Gamma-8)\right] U_{1}^{(0)} \\
& -\frac{1}{10} T S\left[\nabla_{i_{1}} \cdots \nabla_{i_{4}} L^{*} U_{0}^{(0)}+\frac{R}{6} \nabla_{i_{1}} \cdots \nabla_{i_{4}} U_{0}^{(0)}\right] \\
= & I_{i_{1} \cdots i_{4}}^{(0)}-\frac{1}{12} \operatorname{RTS}\left(\nabla_{i_{1}} \cdots \nabla_{i_{4}} U_{0}^{(0)}\right)
\end{aligned}
$$

where

$$
\begin{aligned}
I_{i_{1} \cdots i_{4}}^{(0)} & =-\frac{1}{10} T S\left(\nabla_{i_{1}} \cdots \nabla_{i_{4}} L^{*} U_{0}^{(0)}\right) \\
& =\frac{1}{252} T S\left(9 \nabla^{a} C_{i_{1} i_{2}}^{b}{ }^{c} \nabla_{a} C_{b i_{3} i_{4} c}+4 R C_{i_{1} i_{2}}^{a}{ }^{b} C_{a i_{3} i_{4} b}\right)
\end{aligned}
$$

is the moment of order 4 with respect to $L^{*}: \Lambda^{0} \rightarrow \Lambda^{0}$ (see $\left.[19,21,22]\right)$. Now, $(3.28)$, (3.29) and (3.18) imply

$$
\begin{aligned}
T S & \left(\nabla_{i_{1}} \cdots \nabla_{i_{4}} T^{(0)}\right) \\
& =\frac{1 \cdot}{2^{2} \cdot 3^{2} \cdot 5 \cdot 7} T S\left(45 \nabla^{a} C^{b}{ }_{i_{1} i_{2}}{ }^{c} \nabla_{a} C_{b i_{3} i_{4} c}+13 R C^{a}{ }_{i_{1} i_{2}}{ }^{b} C_{a i_{3} i_{4} b}\right)=0 .
\end{aligned}
$$

Consequently, because of Proposition 3.9, one has $C_{a b c d}=0$

Summarising the results of Propositions $3.4-3.7$ and of Corollaries 3.1 and 3.3 we obtain the following

Proposition 3.10. For $L^{(p)}: \Lambda_{\delta}^{p} \rightarrow \Lambda_{\delta}^{p}(p=1,2)$ to be a Huygens' operator it is necessary that

(i) $\nabla_{a} R=0$

(ii) $g$ is conformally flat or a plañe wave metric.

Because of Proposition 3.3 it remains to investigate the case $C_{a b c d}=0$.

\section{Conformally flat space-times}

In this section we assume $C_{a b c d}=0$. Our aim is the determination of all Huygens' conformally flat metrics with respect to the operator $L^{(p)}$. To this end we employ the "two-component" spinor calculus $[5,11,15,19]$. Let $\sigma^{a}{ }_{A \dot{X}}$ be the complex connection quantities, $\varepsilon_{A B}$ the Levi-Civita spinor and $\Phi_{A B \dot{X} \dot{Y}}$ the spinor equivalent of the tensor $\frac{1}{2} T S\left(R_{a b}\right)$.

It is useful to introduce a spinor dyad $\left\{\kappa_{A}, \mu_{A}\right\}$ satisfying $\kappa_{A} \mu^{A}=1$ (see $\left.[11,20]\right)$. Associated to the spinor dyad there is a null tetrad $\left\{l^{a}, n^{a}, m^{a}, \bar{m}^{a}\right\}$ defined by

$$
l^{a}=\sigma^{a}{ }_{A} \dot{X}^{\kappa^{A}} \bar{\kappa}^{\dot{X}}, \quad n^{a}=\sigma^{a}{ }_{A} \dot{X}^{\mu^{A}} \bar{\mu}^{\dot{X}}, \quad m^{a}=\sigma^{a}{ }_{A} \dot{X}^{A} \bar{\mu}^{\dot{X}} .
$$


The metric tensor can be expressed in terms of the null tetrad by (see [11])

$$
g_{a b}=2\left(l_{(a} n_{b)}-m_{(a} \bar{m}_{b)}\right)
$$

In particular, the spinor equivalent to a bivektor $F_{a b}=F_{[a b]}$ is given by (see [15])

$$
F_{A B \dot{A} \dot{B}}=\varepsilon_{\dot{A} \dot{B}} \phi_{A B}+\varepsilon_{A B} \bar{\phi}_{\dot{A} \dot{B}}
$$

where

$$
\phi_{A B}=\phi_{(A B)}=\frac{1}{2} F_{A B \dot{X}} \dot{x} .
$$

Then the spinor equivalent of the dual $F_{a b}^{*}$ is given by

$$
F_{A B \dot{A} \dot{B}}^{*}=-i\left(\varepsilon_{\dot{A} \dot{B}} \phi_{A B}-\varepsilon_{A B} \bar{\phi}_{\dot{A} \dot{B}}\right)
$$

Furthermore, we have (see [14])

$$
\nabla_{[a} F_{b c]}=0 \quad \Longleftrightarrow \quad \nabla^{a} F_{a b}^{*}=0
$$

Using (3.18), (3.22), (3.10), (3.23) and the spinor calculus, especially the relations (4.3) - (4.5), we obtain after a extensive calculation the following result:

\section{Proposition 4.1.}

(i) The condition

$$
\nabla_{i_{1}}\left(d_{(x)} T^{(1)}\right)_{a_{1} a_{2} \alpha} \equiv \nabla_{i_{1}} \nabla_{\left[a_{1}\right.} T_{\left.a_{2}\right] \alpha}^{(1)}=0
$$

is equivalent to

$$
\begin{aligned}
R\left[T S\left(R_{a b}\right)\right] & =0 \\
T S\left[T S\left(R_{a}{ }^{k}\right) T S\left(R_{b k}\right)\right] & =0 .
\end{aligned}
$$

(ii) The condition

$$
\nabla_{i_{1}}\left(d_{(x)} T^{(2)}\right)_{a_{1} a_{2} a_{3} \alpha_{1} \alpha_{2}} \equiv \nabla_{i_{1}} \nabla_{\left[a_{1}\right.} T_{\left.a_{2} a_{3}\right] \alpha_{1} \alpha_{2}}^{(2)}=0
$$

is equivalent to

$$
R\left[T S\left(R_{a b}\right)\right]=0 .
$$

Remark 4.1. The spinor equivalent to the second equation in (4.6) is given by

$$
\phi_{K \dot{K} \dot{A}(A} \phi_{B) \dot{B}} \dot{K}^{\dot{K}}=0 .
$$

Proposition 4.2. From $R=0$ and (4.8) it follows there exists a real function $\sigma$ and a spinor dyad $\left\{\kappa_{A}, \mu_{A}\right\}$ such that $\Phi_{A \dot{A} B \dot{B}}$ has one of the forms

$$
\phi_{A \dot{A} B \dot{B}}=\sigma \kappa_{A} \kappa_{B} \bar{\kappa}_{\dot{A}} \bar{\kappa}_{\dot{B}}
$$


or

$$
\phi_{A \dot{A} B \dot{B}}=\sigma \kappa_{\left(A \mu_{B)}\right.} \bar{\kappa}_{(\dot{A}} \bar{\mu}_{\dot{B})} .
$$

Proof. If $\left\{l^{a}, n^{a}, m^{a}, \bar{m}^{a}\right\}$ is a complex null tetrad and $m^{a}=\frac{1}{\sqrt{2}}\left(x^{a}+i y^{a}\right)$, then $\left\{l^{a}, n^{a}, x^{a}, y^{a}\right\}$ is a real null tetrad. By means of the classification theory of the Ricci tensor (see $[8,11]$ ) it is easy to show that $T S\left(R_{a b}\right)$ has the canonical form

( $\left.\alpha) T S\left(R_{a b}\right)=2 \sigma_{0} l_{(a} n_{b}\right)+\sigma_{1}\left(l_{a} l_{b}+\varepsilon n_{a} n_{b}\right)+\sigma_{2} x_{a} x_{b}+\sigma_{3} y_{a} y_{b}$

or

( $\left.\beta) T S\left(R_{a b}\right)=2 \sigma_{0}^{\prime} l_{(a} n_{b)}+2 l_{(a} x_{b}\right)+\sigma_{0}^{\prime}\left(x_{a} x_{b}+y_{a} y_{b}\right)$

where $\varepsilon \in\{1,-1,0\}$ and $2 \sigma_{0}-\sigma_{2}-\sigma_{3}=0$. The condition $\operatorname{TS}\left[T S\left(R_{a}^{k}\right) \operatorname{TS}\left(R_{b k}\right)\right]=0$ implies one of the forms

(a) $T S\left(R_{a b}\right)=\sigma l_{a} l_{b}$

(b) $T S\left(R_{a b}\right)=\sigma\left(4 l_{(a} n_{b)}-g_{a b}\right)$

(c) $T S\left(R_{a b}\right)=\sigma\left(l_{a} l_{b}+n_{a} n_{b} \pm m_{a} m_{b} \pm \bar{m}_{a} \bar{m}_{b}\right)$.

Using the relations (4.1), we obtain the result for the cases (a) and (b). In the case (c) we have

$$
\phi_{A \dot{A} B \dot{B}}=\sigma\left[\left(\kappa_{A}+\gamma \mu_{A}\right)\left(\kappa_{B}-\gamma \mu_{B}\right)\left(\bar{\kappa}_{\dot{A}}+\bar{\gamma} \bar{\mu}_{\dot{A}}\right)\left(\bar{\kappa}_{\dot{B}}-\bar{\gamma} \bar{\mu}_{\dot{B}}\right)\right]
$$

with $\gamma \in\{1, i\}$. Putting

$$
\kappa_{A}^{\prime}=\kappa_{A}+\gamma \mu_{A} \quad \text { and } \quad \mu_{A}^{\prime}=-\frac{1}{2 \gamma}\left(\kappa_{A}-\gamma \mu_{A}\right)
$$

we get $\kappa_{A}^{\prime} \mu^{\prime A}=1$ and obtain the representation (ii)

Proposition 4.3. The conditions

$$
\begin{aligned}
& \Phi_{A B \dot{A} \dot{B}}=\sigma \kappa_{\left(A \mu_{B)} \bar{\kappa}_{(\dot{A}} \bar{\mu}_{\dot{B})}\right.} \\
& \nabla_{[a} R_{b] c}=0
\end{aligned}
$$

imply $\nabla_{a} R_{b c}=0$ [20])

Proof. On account of $\kappa_{A} \mu^{A}=1$ there are spinors $A_{A \dot{X}}, B_{A \dot{X}}$ and $C_{A \dot{X}}$ with (see

$$
\begin{aligned}
& \nabla_{A \dot{X}} \kappa_{B}=A_{A \dot{X}} \kappa_{B}+B_{A \dot{X}} \mu_{B} \\
& \nabla_{A \dot{X}} \mu_{B}=C_{A \dot{X}} \kappa_{B}-A_{A \dot{X}} \mu_{B} .
\end{aligned}
$$

The spinor equivalent to $\nabla_{[a} R_{b] c}=0$ is given by $\nabla_{A}^{\dot{Y}} \phi_{B C \dot{X} \dot{Y}}=0$. Consequently, one 
obtains for $\sigma \neq 0$

$$
\begin{aligned}
0= & \frac{2}{\sigma} \nabla{ }_{A}^{\dot{Y}} \phi_{B C \dot{X} \dot{Y}} \\
= & \left(\frac{1}{\sigma} \nabla_{A}^{\dot{Y}} \sigma \bar{\mu}_{\dot{Y}}+2 \bar{C}_{A}^{\dot{Y}} \bar{\kappa}_{\dot{Y}}\right) \kappa_{\left(B \mu_{C}\right)} \bar{\kappa}_{\dot{X}} \\
& +\left(\frac{1}{\sigma} \nabla_{A}^{\dot{Y}} \sigma \bar{\kappa}_{\dot{Y}}+2 \bar{B}_{A}^{\dot{Y}} \bar{\mu}_{\dot{Y}}\right) \kappa_{(B} \mu_{C)} \bar{\mu}_{\dot{X}} \\
& +\left(C{ }_{A}^{\dot{Y}} \bar{\mu}_{\dot{Y}}\right) \kappa_{B} \kappa_{C} \bar{\kappa}_{\dot{X}}+\left(C{ }_{A}^{\dot{Y}} \bar{\kappa}_{\dot{Y}}\right) \kappa_{B} \kappa_{C} \bar{\mu}_{\dot{X}} \\
& +\left(B_{A}^{\dot{Y}} \bar{\mu}_{\dot{Y}}\right) \mu_{B} \mu_{C} \bar{\kappa}_{\dot{X}}+\left(B_{A}^{\dot{Y}} \bar{\kappa}_{\dot{Y}}\right) \mu_{B} \mu_{C} \bar{\mu}_{\dot{X}}
\end{aligned}
$$

hence $\nabla_{A \dot{X}} \sigma=B_{A \dot{X}}=C_{A \dot{X}}=0$ and the assertion is proved

Corollary 4.1. A metric with the properties

$$
R=0, \quad C_{a b c d}=0, \quad \phi_{A B \dot{A} \dot{B}}=\sigma \kappa_{(A} \mu_{B)} \bar{\kappa}_{(\dot{A}} \bar{\mu}_{\dot{B})}
$$

is flat.

Proof. From Proposition 4.2 it follows that $(M, g)$ is symmetric. A symmetric space-time with $R=0$ and $C_{a b c d}=0$ is flat (see $\left.[6,20]\right)$

In [14] there was proved the following

Proposition 4.4. A metric with the properties

$$
R=0, \quad C_{a b c d}=0, \quad \phi_{A B \dot{A} \dot{B}}=\sigma \kappa_{A} \kappa_{B} \bar{\kappa}_{\dot{A}} \bar{\kappa}_{\dot{B}}
$$

is a plane wave metric.

Propositions 4.1 - 4.4 and Corollary 4.1 imply the following

Corollary 4.2. A conformally flat metric with $d_{(x)} T^{(1)}(x, y)=0$ and $\nabla_{a} R=0$ is either a plane wave metric or a metric with $T S\left(R_{a b}\right)=0$.

The following proposition is a consequence of the relation $T^{(p)}(x, y)=A(p) \gamma^{(p)}(x, y)$ with $A(p)=$ const and $d_{(x)} \gamma^{(p)}=0$, which was proved for space-times of constant curvature in $[1,3]$.

Proposition 4.5. In a space-time of constant curvature one has

$$
d_{(x)} T^{(p)}(x, y)=0 \quad(p=1,2) .
$$

Finally, we prove Theorem 1.1. 


\section{Corollary 4.3.}

(i) $L^{(1)}: \Lambda_{\delta}^{1} \rightarrow \Lambda_{\delta}^{1}$ is a Huygens' operator if and only if $g$ is either a plane wave metric or a metric with $C_{a b c d}=0$ and $R_{a b}=\frac{1}{4} R g_{a b}$.

(ii) $L^{(2)}: \Lambda_{\delta}^{2} \rightarrow \Lambda_{\delta}^{2}$ is a Huygens' operator if and only if $g$ is either a plane wave metric or a metric with $C_{a b c d}=0$ and $R\left(R_{a b}-\frac{1}{4} R g_{a b)}=0\right.$.

Proof. If $g$ is a plane wave metric or a metric of constant curvature, then

$$
d_{(x)} T^{(p)}(x, y)=0
$$

i.e. $L^{(p)}$ is a Huygens' operator (Propositions 3.3 and 4.5). If $C_{a b c d}=0$ and $R=0$, then $T^{(2)}(x, y)=0$ (Propositions 3.1 and 3.2). Consequently, $d_{(x)} T^{(2)}(x, y)=0$.

Conversely, if $L^{(p)}(p=1,2)$ is a Huygens' operator, then $\mathrm{g}$ is a plane wave metric or conformally flat with $\nabla_{a} R=0$ (Proposition 3.10). The assertion (i) follows from Collorary 4.2. Finally, Proposition 4.1 implies $R\left(R_{a b}-\frac{1}{4} R g_{a b}\right)=0$

\section{References}

[1] Belger, M.: Geodätische Formen auf pseudo-Riemannschen Räumen. Serdica Bulg. Math. Publ. 4 (1978), 43 - 49.

[2] Friedlander, F. G.: The Wave Equation on a Curved Space-Time. Cambridge: University Press 1975.

[3] Günther, P.: Harmonische geodätische p-Formen in nichteuklidischen Räumen. Math. Nachr. 28 (1965), 291 - 304.

[4] Günther, P.: Ein Beispiel einer nichttrivialen Huygensschen Differentialgleichung mit vier unabhängigen Veränderlichen. Arch. Rat. Mech. Anal. 18 (1965), 103 - 106.

[5] Günther, P.: Huygen's Principle and Hyperbolic Equations. Boston: Academic Press 1988.

[6] Günther, P. and V. Wünsch: Maxwellsche Gleichungen und Huygenssches Prinzip. Math. Nachr. 63 (1974), 97 - 121.

[7] Hadamard, J.: Lectures on Cauchy's Problem in Linear Partial Differential Equations. New Haven: Yale University Press 1923.

[8] Hall, G. S.: The classification of the Ricci tensor in general relativity theory. J. Phys. A9 (1976), $541-557$.

[9] Helgason, S.: Huygens' principle for wave equations on symmetric spaces. J. Funkt. Anal. 107 (1992), $279-288$.

[10] Illge, R.: Zur Gültigkeit des Huygensschen Prinzips bei hyperbolischen Differentialgleichungssystemen in statischen Raum-Zeiten. Z. Anal. Anw. 6 (1987), $385-407$.

[11] Kramer, D., Stephani, H., MacCallum, M. A. H. and E. Herlt: Exact Solutions of Einstein's Equations. Berlin: Dt. Verl. Wiss. 1980.

[12] Lichnerowicz, A.: Chaps spinoriels et propagateurs en relativité générale. Bull. Soc. Math. France 92 (1964), $11-100$.

[13] McLenaghan, R. G.: Huygen's Principle. Ann. Inst. H. Poincaré A37 (1982), 211 - 236. 
[14] McLenaghan, R. G., Tariq, N. and B. O. Tupper: Conformally flat solutions of the Einstein-Maxwell equations for null electromagnetic fields. J. Math. Phys. 16 (1975), $829-841$.

[15] Penrose, R. and W. Rindler: Spinors and Space-Time. Vol. 1 and 2. Cambridge: University Press 1984 and 1986.

[16] Rinke, B. and V. Wünsch: Zum Huygensschen Prinzip bei der skalaren Wellengleichung. Beitr. Anal. 18 (1981), $43-75$.

[17] Schimming, R.: Zur Gültigkeit des Huygensschen Prinzips bei einer speziellen Metrik. Z. Angew. Math. Mech. (ZAMM) 51 (1971), $202-208$.

[18] Schimming, R.: Das Huygenssche Prinzip bei hyperbolischen Differentialgleichungen für allgemeine Felder. Beitr. Anal. 11 (1978), $45-90$.

[19] Wünsch, V.: Cauchy-Problem und Huygenssches Prinzip bei einigen Klassen spinorieller Feldgleichungen. Parts I und II. Beitr. Anal. 12 (1978), $47-76$ and 13 (1979), $147-177$.

[20] Wünsch, V.: Charakterisierung von Raum-Zeit-Mannigfaltigkeiten durch Relationen zwischen ihren Krümmungsspinorer, unter Benutzung eines modifizierten Newman-PenroseKalküls. Math. Nachr. 89 (1979), 321 - 336.

[21] Wünsch, V.: C-Räume und Huygenssches Prinzip. Wiss. Z. Päd. Hochschule ErfurtMühlhausen, Math.-Nat. Reihe 23 (1987)1, 103 - 111.

[22] Wünsch, V.: Huygens' principle on Petrov type $N$ space-times. Ann. Inst. H. Poincaré A60 (1994), $87-102$.

Received 29.05.1996 